\title{
The maximum spin of Millisecond Pulsar and the gravitational wave
}

\author{
C. M. Zhang \\ National Astronomical Observatories, Chinese Academy of Sciences, Beijing 100012, China \\ email: zhangcm@bao.ac.cn
}

\begin{abstract}
The gravitational wave radiation will release the energy momentum, which will dissipate the rotation of neutron star while in the accretion process. If the deformation of star is known, then we can estimate the maximum spin frequency of pulsar, based on which we can interpret why the spin periods of all millisecond pulsars cannot be less than one millisecond.
\end{abstract}

Keywords. neutron star, pulsar, millisecond pulsar, gravitational waves

\section{Gravitational wave induced spin limit of millisecond pulsar}

The fastest spin frequency of millisecond pulsar (MSP) has been observed to be $1.4 \mathrm{~ms}$, and the sub-millisecond pulsar has been expected by astronomers, since such a fast spin compact object could constrain its radius and mass and even EOS of compact object. However, why do we have not yet observed the MSPs with the period less than $1.4 \mathrm{~ms}$ ? If there exists somewhat mechanism to curb the spin velocity of MSP? In this short paper, we investigate a way of producing a pulsar spin of some milliseconds by the dissipating the star rotating energy on account of the star deformation induced gravitational wave. The main idea is introduced in the following. In the formation stage of MSP, its magnetic field is also decayed (Alpar et al. 1982; Bhattacharya \& van den Heuvel 1991; Zhang \& Kojima 2006), so the calculation of spin-up of star should also consider its field decay, which has been performed (Wang et al. 2011) by exploiting the spin-up formula of Ghosh $\&$ Lamb (1979). Figure 1 show the calculations with various accretion rates, where we find that evolution curves can cover the observed points shown in the figure, then the spin can reach millisecond.

The spin of MSP is produced by the accretion of companion in the binary system, the spin can be spun-up to sub-millisecond if the star accretes $0.2 M_{\odot}$.

Then, in the accretion process, if the gravitational radiation occurs by the deformation of neutron star, the MSP spin may be limited. This idea has been mentioned by Bildsten (1998) and Chakrabarty (2005). Therefore, we consider this effect, and estimate its influence on the MSP spin. If NS has a deformation efficiency of $\epsilon$, then its gravitational wave emission power is

$$
\dot{E}_{G W}=\frac{32}{5} \frac{G}{c^{5}} I^{2} \varepsilon^{2} \Omega^{6},
$$

which arises as a kinetic energy loss. The accretion spin-up rate and GW emission rate should be balanced if both are same.

$$
\dot{E}_{G W}=I \Omega \dot{\Omega} \propto \Omega^{6}
$$

In the binary systems of accreting NSs, the accretion accelerate rate is given by conservation of momentum 


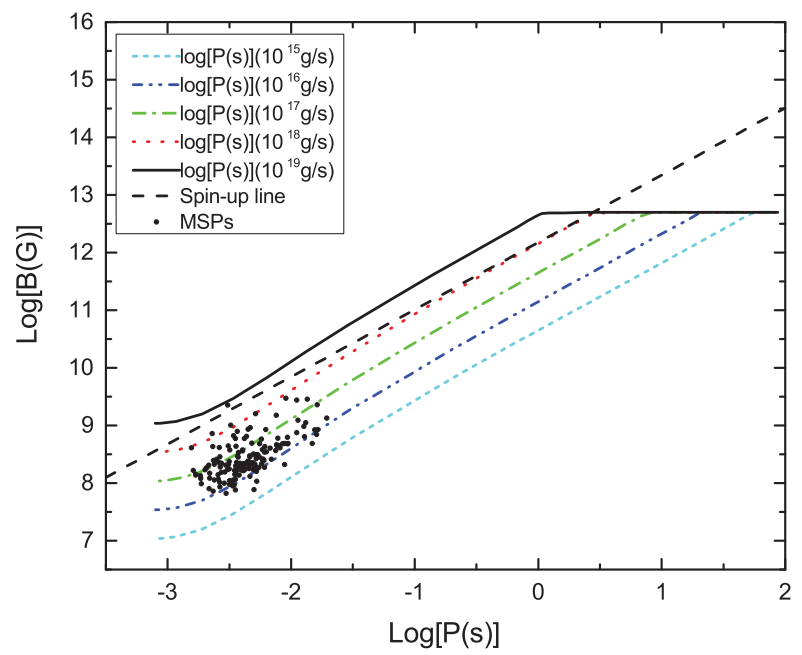

Figure 1. Magnetic field and spin period diagram, where the plotted lines from the above to bottom stand for the accretion spin-up without gravitational wave, with the accretion rates from the super-Eddington rate $10^{19} \mathrm{~g} \mathrm{~s}^{-1}$ to $10^{15} \mathrm{~g} \mathrm{~s}^{-1}$.

$$
I \dot{\Omega}=\dot{M} R_{M}{ }^{2} \sqrt{\frac{G M}{R_{M}^{3}}}=\dot{M} \sqrt{G M R_{M}}=\dot{M} \sqrt{\frac{2 G M}{c^{2}} \frac{c^{2} R_{M}}{2}}=\dot{M} c \sqrt{\frac{R_{s} R_{M}}{2}}
$$

where $R_{s}=2 G M / c^{2}=3 \mathrm{~km}\left(M / M_{\odot}\right)$ is the Schwarzschild radius, we take $M / M_{\odot}=1.4$, and $R_{M}$ is magnetosphere radius (ZK06). When the deceleration caused by gravitational wave equal to accretion acceleration rate, the rotation velocity of NSs will stop at some value.

\section{Main conclusions}

The maximum spin frequency of NS can be $0.8 \mathrm{~ms}$ in theory, while the fastest MSP observed by now is $1.4 \mathrm{~ms}$. We try to ascribe the GW effect to the limit of MSP spin. In the accretion process, the accretion materials may not distribute uniformly, and the strong magnetic region may accumulate more matter than that of low filed polar region. This slightly deformation of MSP structure can cause a GW, which limit the fastest spin. From observation, we cannot sure this deformation, and may detect the second derivative of spin to determine the braking index of NS to know the existence of GW emission.

\section{References}

Alpar, M. A., Cheng, A. F., Ruderman, M. A., \& Shaham, J. 1982, Nature, 300, 728

Bhattacharya, D. \& van den Heuvel, E. P. J. 1991, Phys. Rep., 203, 1

Bildsten, L. 1998, ApJ, 501, L89

Chakrabarty, D. 2005, "Millisecond Pulsars in X-Ray Binaries," in Binary Radio Pulsars, eds.

F. A. Rasio \& I. H. Stairs (ASP: San Francisco) p. 279

Ghosh, P. \& Lamb, F. K. 1979, ApJ, 234, 296

Wang, J., Zhang, C. M., Zhao, Y. H., et al. 2011, A\& A, 526, A88

Zhang, C. M. \& Kojima, Y. 2006, MNRAS, 366, 137 (ZK06) 